Article

\title{
Tartary Buckwheat Transcription Factor FtbZIP5, Regulated by FtSnRK2.6, Can Improve Salt/Drought Resistance in Transgenic Arabidopsis
}

\author{
Qi Li ${ }^{\dagger}$, Haixia Zhao ${ }^{\dagger}$, Xiaoli Wang, Jingyue Kang, Bingbing Lv, Qixin Dong, Chenglei Li, \\ Hui Chen and Qi Wu* \\ College of Life Science, Sichuan Agricultural University, No. 46, Xinkang Road, Ya'an 625014, China; \\ lq760621985@gmail.com (Q.L.); zhaohaixia@sicau.edu.cn (H.Z.); wangxiaoli288@163.com (X.W.); \\ kangjingyue2020@163.com (J.K.); lvbing1228@163.com (B.L.); dqxczwq00@163.com (Q.D.); \\ lichenlei@sicau.edu.cn (C.L.); chenhui@sicau.edu.cn (H.C.) \\ * Correspondence: wuqi@sicau.edu.cn \\ † These authors contributed equally to this work.
}

Received: 23 December 2019; Accepted: 5 February 2020; Published: 7 February 2020

\begin{abstract}
ZIP transcription factors have been reported to be involved in many different biological processes in plants. The ABA (abscisic acid)-dependent AREB/ABF-SnRK2 pathway has been shown to play a key role in the response to osmotic stress in model plants. In this study, a novel bZIP gene, FtbZIP5, was isolated from tartary buckwheat, and its role in the response to drought and salt stress was characterized by transgenic Arabidopsis. We found that FtbZIP5 has transcriptional activation activity, which is located in the nucleus and specifically binds to ABRE elements. It can be induced by exposure to PEG6000, salt and ABA in tartary buckwheat. The ectopic expression of FtbZIP5 reduced the sensitivity of transgenic plants to drought and high salt levels and reduced the oxidative damage in plants by regulating the antioxidant system at a physiological level. In addition, we found that, under drought and salt stress, the expression levels of several ABA-dependent stress response genes (RD29A, RD29B, RAB18, RD26, RD20 and COR15) in the transgenic plants increased significantly compared with their expression levels in the wild type plants. Ectopic expression of FtbZIP5 in Arabidopsis can partially complement the function of the ABA-insensitive mutant abi5-1 (abscisic acid-insensitive 5-1). Moreover, we screened FtSnRK2.6, which might phosphorylate FtbZIP5, in a yeast two-hybrid experiment. Taken together, these results suggest that FtbZIP5, as a positive regulator, mediates plant tolerance to salt and drought through ABA-dependent signaling pathways.
\end{abstract}

Keywords: tartary buckwheat; AREB/ABF; salt and drought stress

\section{Introduction}

Exposure to environmental stresses such as drought and salt can adversely affect plant growth and crop yield [1]. The phytohormone abscisic acid (ABA), as a component of signaling, is widely involved in the developmental process in plants and responds to environmental stimuli [2,3]. Endogenous ABA increases in plants under osmotic stress, and many genes are activated by the ABA response element (ABRE) in the promoter region [4,5]. Currently, the SnRK2-AREB/ABF pathway is considered to govern major ABA-mediated ABRE-dependent gene expression in response to osmotic stress [6].

Among the large number of bZIP transcription factors identified in Arabidopsis thaliana, ABA signal transduction has been extensively studied in group A, which has 13 members [7,8]. Based on phylogenetic analysis, the bZIP proteins with three conservative N-terminal domains can be divided into two categories [9,10]. The ABI5/AtDPBF family genes (DPBF2/AtbZIP67, DPBF4, AREB3, ABI5 and $E E L)$ are expressed in seeds and play an important role in seed maturation and development [11,12]. 
However, the AREB/ABF family of genes (AREB1/ABF2, AREB2/ABF4, ABF1 and ABF3) are mainly expressed in vegetative tissues under abiotic stress and participate in plant resistance to abiotic stress $[13,14]$. After their discovery, it was found that the functions of AREB1/ABF2, AREB2/ABF4 and ABF3 did not completely overlap and that they could form homodimers or heterodimers under osmotic stress and, in this form, could coordinate the regulation of ABRE-dependent gene expression according to ABA signaling $[15,16]$. Therefore, the functions of AREB/ABF transcription factors show diversity and complexity and have potential for further study.

Studies have shown that AREB/ABF must be activated in an ABA-dependent manner, and the post-transcriptional modification is the phosphorylation by SnRK2 proteins (SNF1-related protein kinases subfamily 2) $[17,18]$. The conserved phosphorylation site RXXS/T in AREB/ABF was identified as the target site of the SnRK2s and other general Ser/Thr kinases $[19,20]$. Furthermore, studies have compared triple mutants of AREB/ABF and subclass III SnRK2s, which are involved in stress resistance in Arabidopsis, and it has been shown that the downstream genes of the ABA-dependent type genes overlap [14]. Loss of SnRK2 protein kinase family function prevents most of the ABA response [21]. In addition, phylogenetic analysis also supports the supposition that SnRK2-AREB/ABF in plants is ubiquitous and part of a major positive regulatory pathway [16,22].

Here, we isolated FtbZIP5 in tartary buckwheat (Fagopyrum tataricum), identified its molecular characteristics and performed functional replenishment experiments in the Arabidopsis mutant abi5-1. Further study confirmed the role of FtbZIP5. Specifically, the physiological and biochemical changes and the expression levels of the downstream stress genes in the transgenic and WT (wild type) plants were compared after stress treatment. Furthermore, we also screened the SnRK2 protein kinases interacting with FtbZIP5. The results may lead to a better understanding of the regulatory network in which FtbZIP5 participates.

\section{Results}

\subsection{Cloning and Molecular Characterization of FtbZIP5}

In this study, FtbZIP5 (GenBank Accession No. MN_120689) was cloned and isolated from tartary buckwheat. The sequence analysis by ExPASy ProtParam on the online website showed that the ORF was composed of 419 amino acids with a molecular weight of $45.671 \mathrm{kDa}$ and an isoelectric point of 8.46. The results of the multiple sequence alignment analysis show that FtbZIP5 has not only a bZIP domain but also potential phosphorylation peptides, $\mathrm{C} 1, \mathrm{C} 2, \mathrm{C} 3$ and $\mathrm{C} 4$, similar to other identified AREB/ABFs (Figure 1A,B). Furthermore, we chose to construct a phylogenetic tree by combining FtbZIP5 with 13 members of the Arabidopsis bZIP transcription factor A subfamily (Figure 1C). The results showed that FtbZIP5 was closely related to four AREB/ABFs involved in stress resistance in Arabidopsis.

\subsection{FtbZIP5 Expression is Involved in the Response to Abiotic Stress}

To further determine whether FtbZIP5 is involved in the abiotic stress signaling pathway, the expression pattern of FtbZIP5 after stress was induced was analyzed by qRT-PCR (Figure 2A-C). After $100 \mu \mathrm{M}$ ABA treatment, the expression level of FtbZIP5 increased rapidly, more than eight-fold, within two hours and then decreased. However, the changes in FtbZIP5 expression levels after the salt and drought treatments were more pronounced than those after the ABA treatment and decreased sharply after peaking at $3 \mathrm{~h}$ and $2 \mathrm{~h}$, respectively. When treated with $150 \mathrm{mM} \mathrm{NaCl}$ and $30 \%$ PEG6000 for $3 \mathrm{~h}$, the transcription level of FtbZIP5 was 35.6-fold and more than 52-fold, respectively. The maximum value (at least eight-fold) was reached after ABA treatment for two hours. The results revealed that FtbZIP5 might be involved in the response of tartary buckwheat to exogenous stress induced by ABA, drought and salt treatments. Through the analysis of the phylogenetic tree and the expression patterns, it was preliminarily determined that FtbZIP5 might be involved in the response to abiotic stress. 
A

NLS

\begin{tabular}{|l|l|l|l|l|l|l|l}
\hline C1 & C2 & C3 & bZIP & C4 \\
\hline
\end{tabular}

C1 PLARQTSIYSLTFDEFQNS C2 YLQRQGSLTLPRTISQKTVD

C3 ATQRQPTLGELTLEELVEA C4 KKRCLRRTQTGVW NLS:EKVI

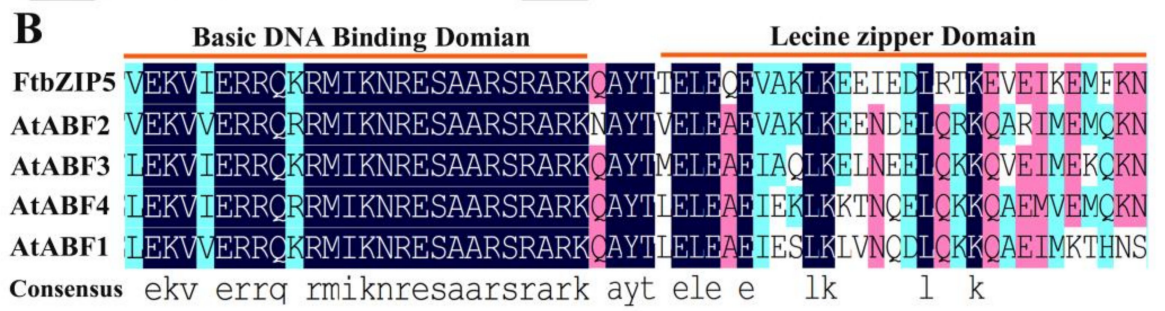

C

ABF1(NM_001198254)

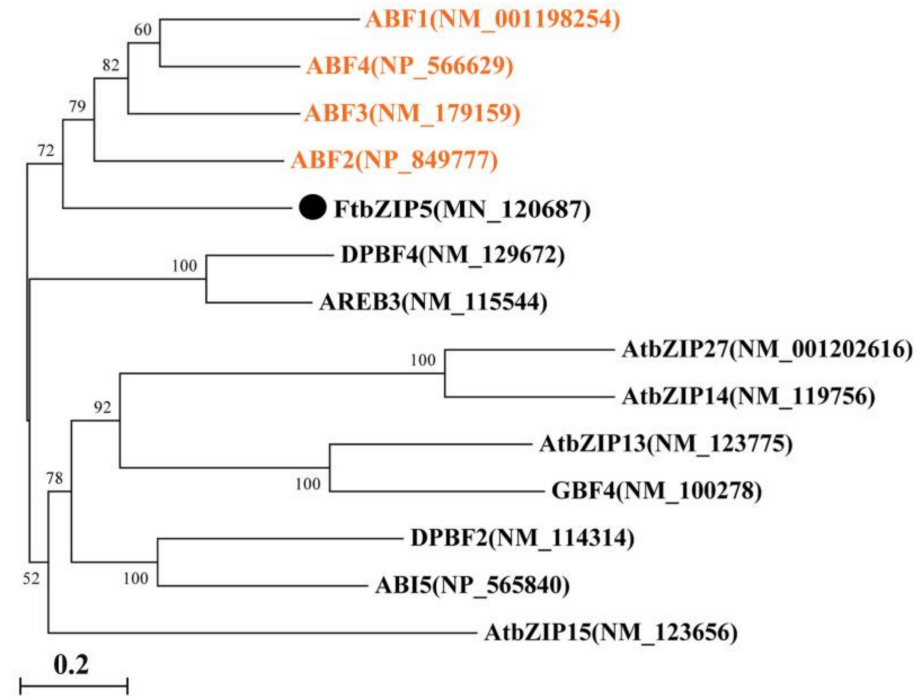

Figure 1. Structure and sequence analysis of FtbZIP5 protein. (A) The conserved domain of FtbZIP5 protein. C1, C2, C3 and C4, four potential phosphorylation sites; NLS, hypothetical nuclear positioning signal. (B) Sequence alignment of four AREB proteins related to stress resistance in Arabidopsis. (C) Results from the phylogenetic analysis of FtbZIP5 and members of subfamily A of bZIP transcription factors in Arabidopsis.
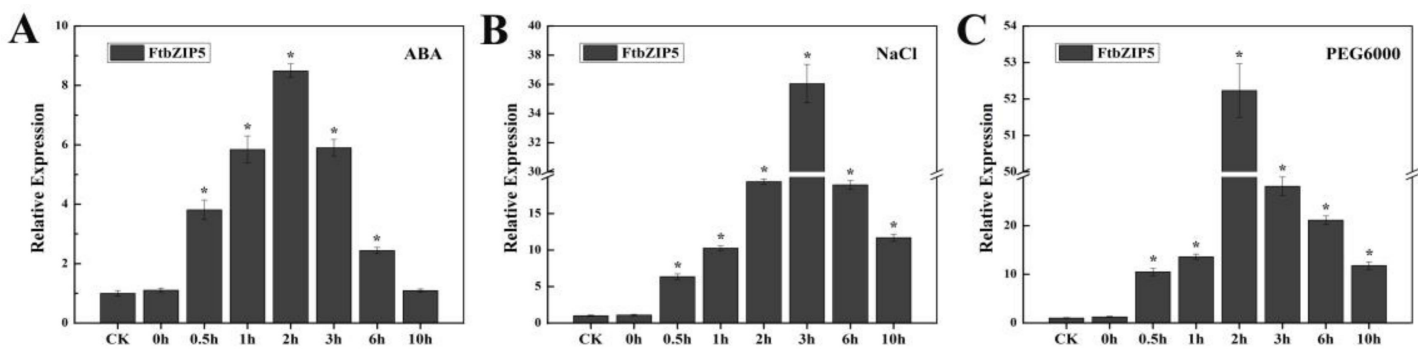

Figure 2. Expression patterns of FtbZIP5 following ABA (A), $\mathrm{NaCl}$ (B) and PEG6000 (C) treatments. The transcriptional abundance of FtbZIP5 in the absence of a stress treatment was used as the CK (control check). Error bars represent $\pm \mathrm{SD}$, and each value represents the averages of three repetitions. Significant difference values were replaced by ${ }^{*}$ to indicate $p<0.05$.

\subsection{Characterization of FtbZIP5 as a Transcription Factor}

To understand the characteristics of FtbZIP5 as a transcription regulator, we determined its localization, transcriptional activity and specific binding elements. As shown in Figure 3A, the yellow 
fluorescent protein carried by FtbZIP5 was localized identically to the blue fluorescence emitted by DAPI. Furthermore, we examined the transcriptional activation of FtbZIP5 in yeast. All transformants were cultured on SD/-His-Trp plates. The negative control (empty yeast and empty yeast + empty vector) could not grow on the double-deficient medium. The positive control (pBridge-FtbHLH2) and pBridge-FtbZIP5 grew normally on the SD/-His-Trp medium (Figure 3B) and was stained blue by $X$ - $\beta$-gal on filter paper (Figure $3 \mathrm{C}$ ). The results showed that FtbZIP5 had transcriptional activity in yeast. As shown in Figure 3D, all the transformants could grow normally in SD/-Trp-Leu, and only the transformants carrying pGADT7-FtbZIP5 + pHIS2.1-3×ABRE could grow on SD/-Trp-Leu-His medium containing $50 \mathrm{mM} 3-\mathrm{AT}$. The results proved that FtbZIP5 could be specifically combined with ABRE in a yeast system.

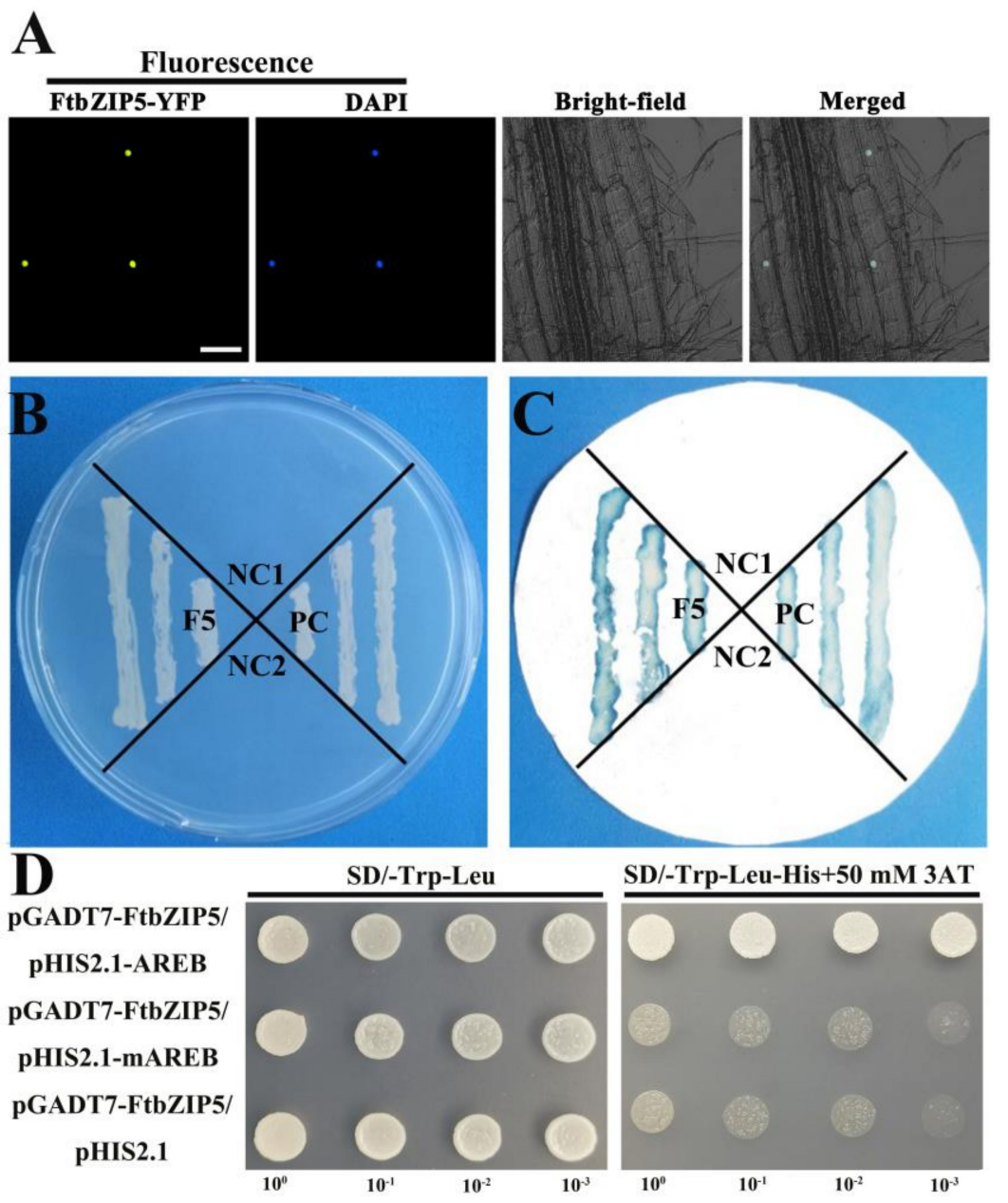

Figure 3. Photographs of FtbZIP5 localization, transcriptional activity and binding to ABRE cis-acting elements. Photographs of FtbZIP5 localization and transcriptional activity experiments. (A) Subcellular localization of FtbZIP5 protein. Scale bars $=100 \mu \mathrm{m}($ B $)$ Culture of the transformed yeast cells on SD/-His-Trp medium. (C) Experimental analysis of galactosidase staining with $x$ - $\beta$-gal on filter paper. NC1:AH109 cells and NC2:pBridge empty plasmids were used as a negative control. PC:pBridge-FtbHLH2 was the positive control used to evaluate the transcriptional activity of pBridge-FtbZIP5. (D) Yeast one hybrid experiment with $3 \times A B R E$ or $3 \times \mathrm{mABRE} 3$ as bait. The yeast cells carrying pGADT7-FtbZIP5 + pHIS2.1-3×ABRE/pHIS2.1-3×mABRE/pHIS2.1 grew on SD/-Trp -Leu and on SD/-Trp-Leu-His containing $50 \mathrm{mM}$ of 3-AT. 
2.4. Ectopic Expression of FtbZIP5 Increased the Sensitivity of Arabidopsis Seeds to Exogenous ABA at the Germination Stage

To explore the effect of ectopic expression of FtbZIP5 during the resistance of transgenic Arabidopsis, we analyzed the germination rate and root elongation of the transgenic lines (L2, L4 and L6) and the WT seeds under adverse conditions. In 1/2-strength MS medium without stress conditions, the germination rates and the root lengths of the transgenic lines and the WT were similar at 7 days. However, in 1/2-strength MS medium containing $0.6 \mu \mathrm{M} \mathrm{ABA}$, the germination rate of the WT seed was at least three-fold that of the transgenic line on the 7th day (Figure 4A,B). In addition, in 1/2-strength MS medium containing $150 \mathrm{mM} \mathrm{NaCl}$ or $200 \mathrm{mM}$ mannitol, the germination rates and root lengths of the transgenic lines were significantly greater than those of the WT. These results showed that the ectopic expression of FtbZIP5 decreased the sensitivity to salt and drought and increased the sensitivity to exogenous ABA.

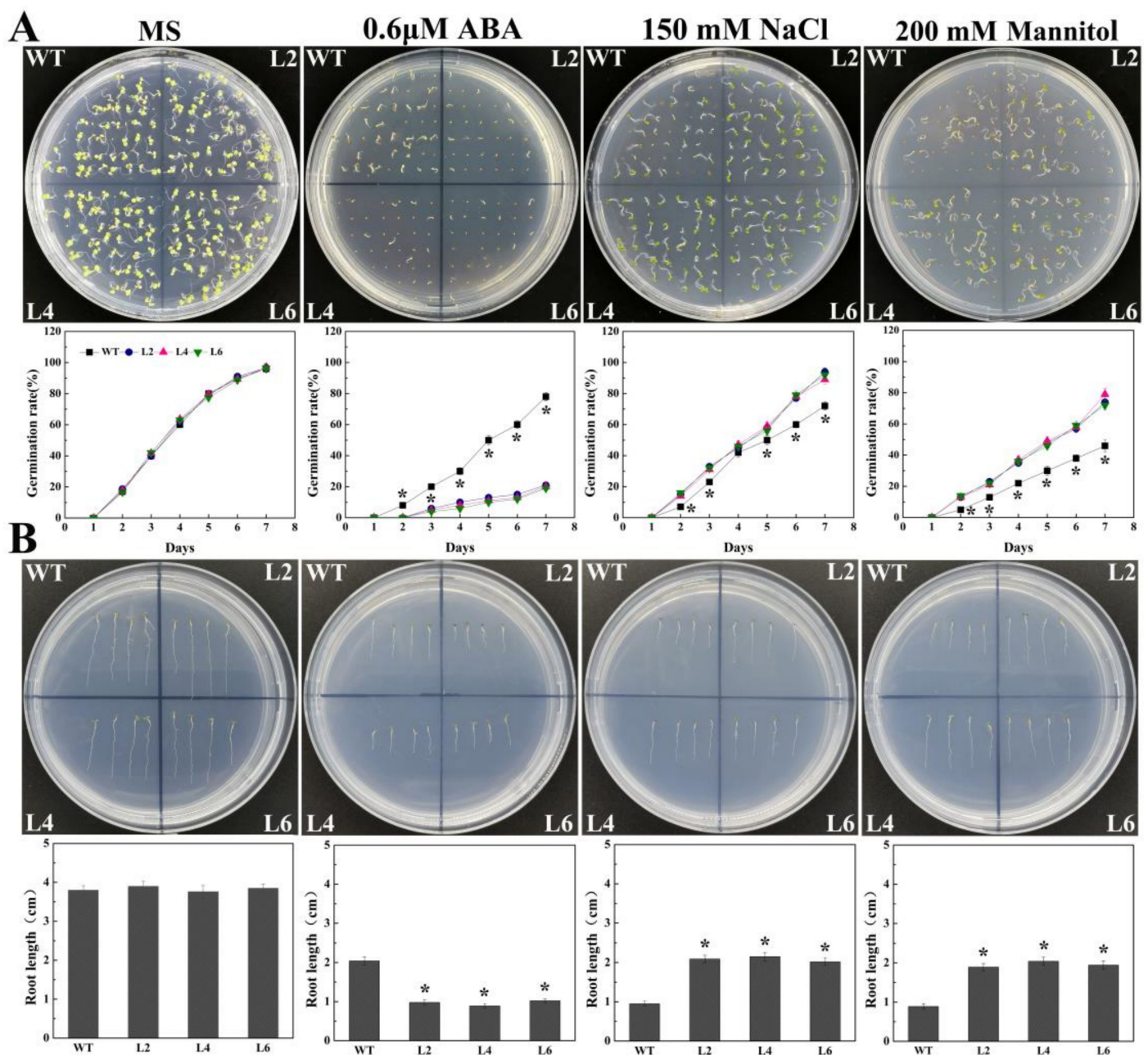

Figure 4. Comparison of the effects of abiotic stress on seed germination and root elongation of WT plants and of the transgenic lines. (A) The germination rate of the WT plants and transgenic line seeds cultured on $0.6 \mu \mathrm{m} \mathrm{ABA,} 150 \mathrm{mM} \mathrm{NaCl}$ and $200 \mathrm{mM}$ mannitol in 1/2-strength MS medium for 7 days. (B) The phenotype and root length of transgenic and WT plants were analyzed on 1/2-strength MS plates with $0.6 \mu \mathrm{m} \mathrm{ABA}, 150 \mathrm{mM} \mathrm{NaCl}$ and $200 \mathrm{mM}$ mannitol for 10 days. The growth of the WT plants and transgenic lines in the $1 / 2$-strength MS plates was used for control values. The means \pm SD represent each value from three replicate experiments. * Indicates a significant difference between the transgenic lines and the WT plants $(p<0.05)$. 


\subsection{Ectopic Expression of FtbZIP5 Enhanced the Resistance of Transgenic Arabidopsis to Drought and Salt Stress}

To confirm the function of FtbZIP5 in Arabidopsis stress resistance, the phenotypic and physiological changes in the transgenic lines and the WT were analyzed after drought, salt and oxidative stress. The initial number of plants used before treatment was 30 plants per pot, and the survival rate of transgenic lines (L2, L4 and L6) after 3 weeks of dewatering and 7 days of rewatering was approximately $60 \%$ higher than that of WT (Figure 5B). In addition, the survival rate of the WT plants was at least $25 \%$ lower than that of the L2, L4 and L6 plants after 3 weeks of salt stress treatment. Furthermore, the levels of the antioxidant enzymes (SOD, POD and CAT) and the proline content in the two-week-old transgenic subjected to drought conditions or treated with salt were more insensitive than those of the WT plants (Figure 5C, Figure 6C, Figure 7C-E), while the content of MDA was more insensitive than that of the WT (Figure 5D, Figure 6D). Consistently, the results showed that the chlorophyll degradation rate in the transgenic lines was lower than that of the WT at MV concentration of $10 \mu \mathrm{M}$ and $30 \mu \mathrm{M}$ MV, as shown in Figure 8A,B.

To investigate whether FtbZIP5 regulates ROS balance in plants, we quantitatively measured ROS $\left(\mathrm{H}_{2} \mathrm{O}_{2}\right.$ and $\left.\mathrm{O}^{-}\right)$accumulation in the L2, L4, L6 and WT leaves by DAB and NBT staining after 10 days of drought or salt treatment. As shown in Figure 7A and 7B, the ROS accumulation in the transgenic plants was significantly lower than that in the WT plants. In conclusion, our results suggest that FtbZIP5 may enhance the stress resistance of transgenic Arabidopsis by regulating the accumulation of ROS and inducing antioxidant systems in plants.
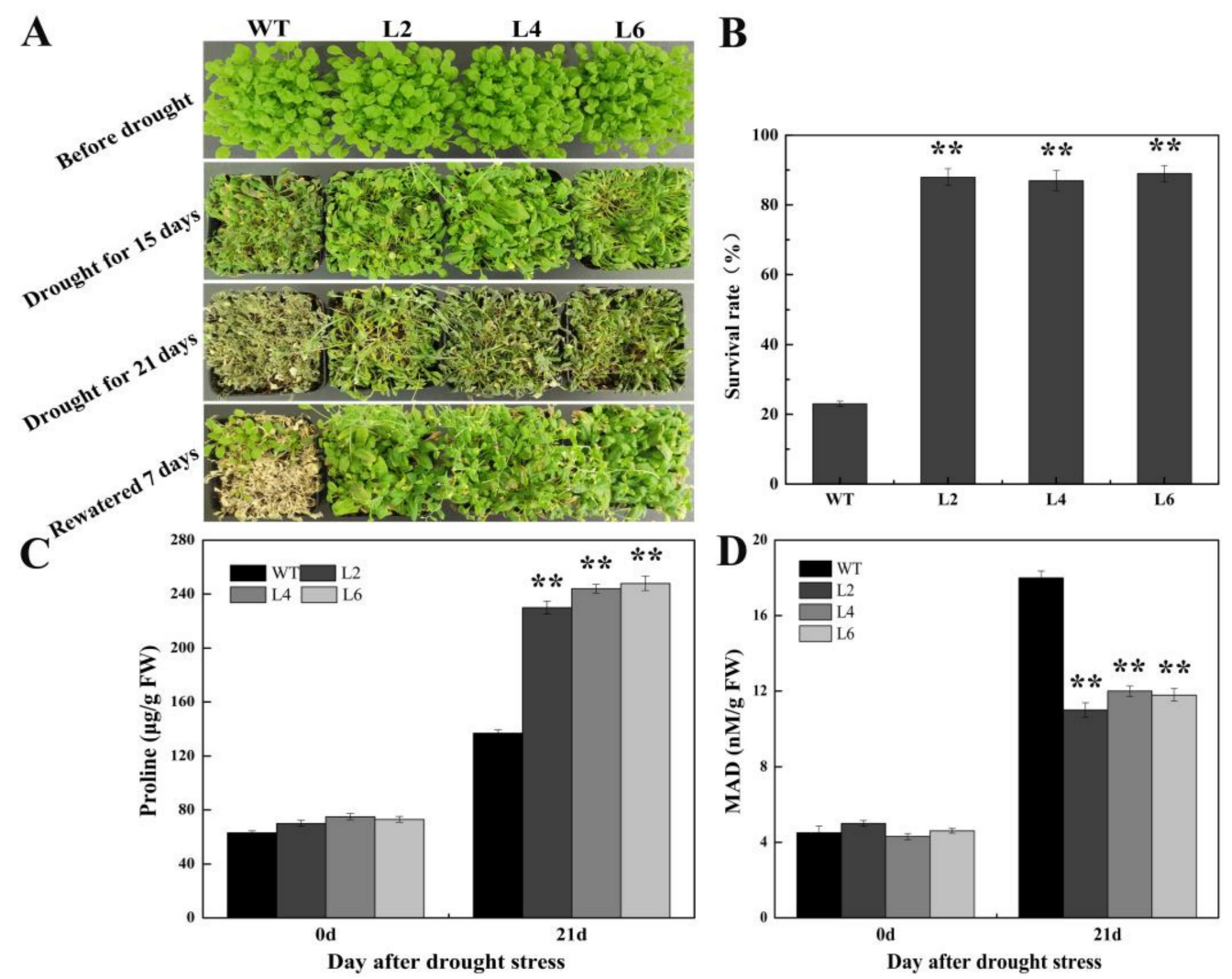

Figure 5. Photographs showing a comparison of the drought resistance state of the transgenic and WT plants. (A) Effects of drought on the transgenic and WT plants. (B) The survival rate of the transgenic and WT plants after being rewatered for 7 days. (C) The proline content. (D) The MDA content. Error bars represent $\pm S D$, and each value represents the averages of three repeated measurements. ${ }^{* *}$ indicate significant differences between the transgenic plants and the WT plants $(p<0.01)$. 

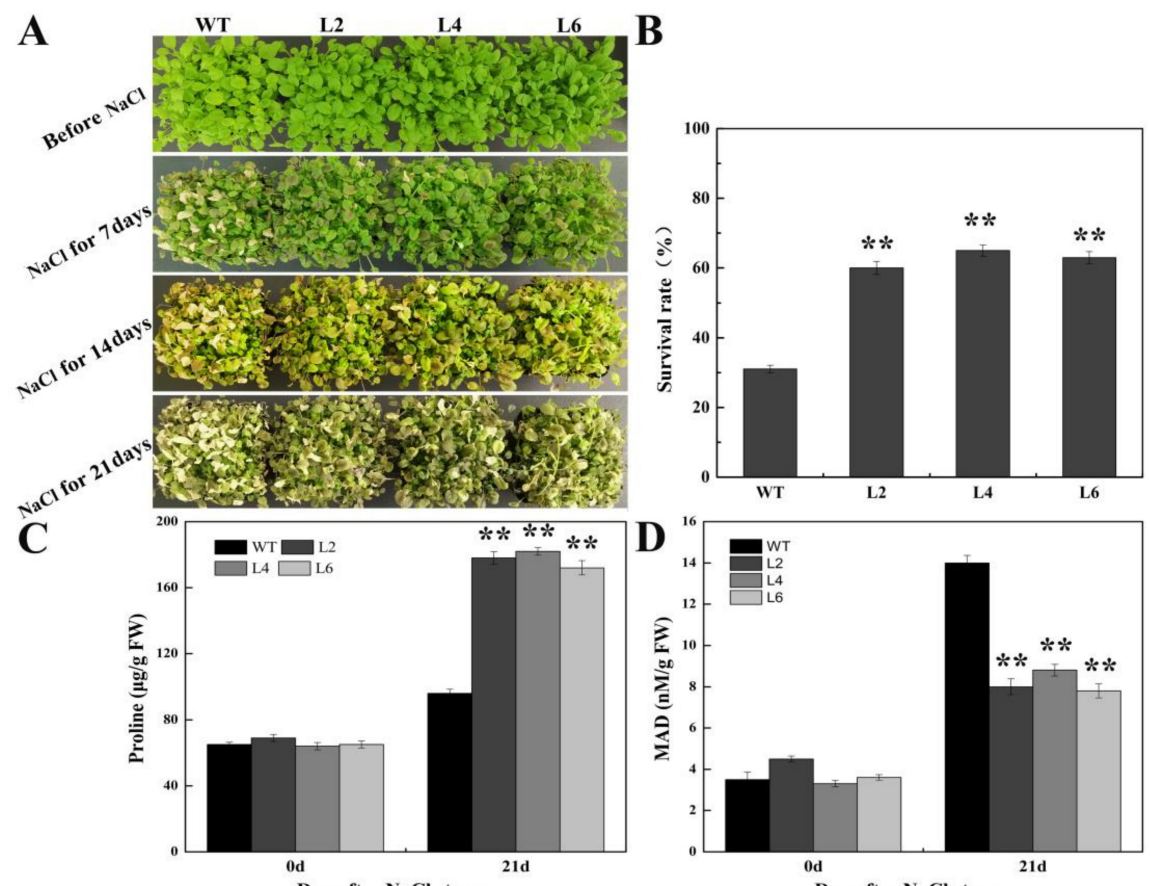

Figure 6. Photographs showing a comparison of the salt tolerance between transgenic and WT plants. (A) Effects of drought on the phenotypes of the L2, L4, L6 and WT plants. (B) The survival rate was calculated 7 days after rehydration. (C) Proline content. (D) MDA content. Error bars represent \pm SD, and each value represents the averages of three repeated measures. ${ }^{* *}$ indicate the significant differences between the L2/L4/L6 plants and the WT plants $(p<0.01)$.

A

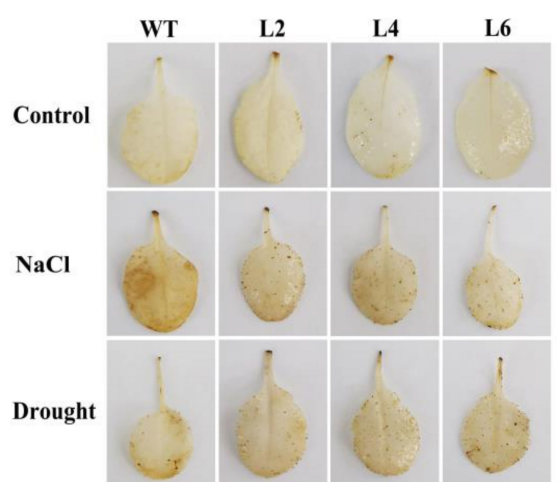

B
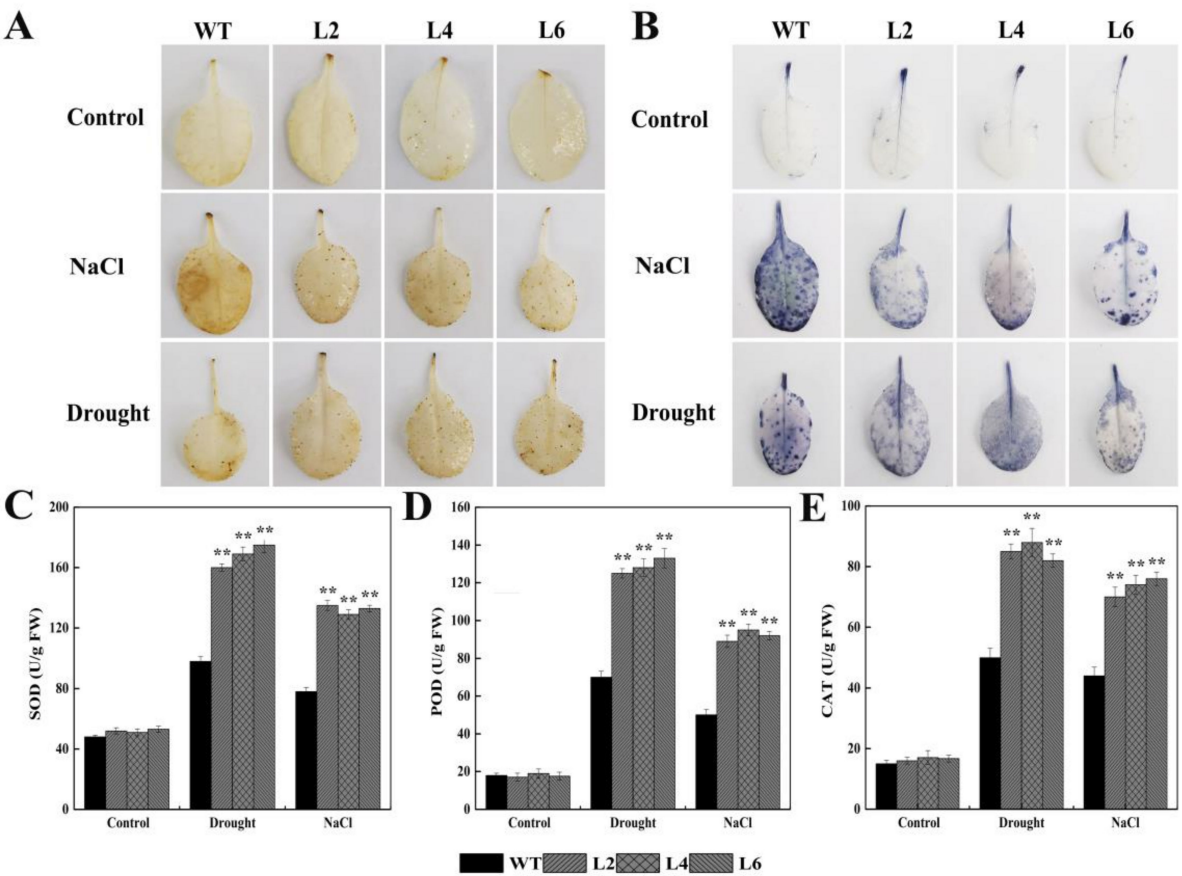

Figure 7. Effects of ectopic expression of FtbZIP5 on ROS accumulation and oxidative damage in Arabidopsis. The accumulation of $\mathrm{H}_{2} \mathrm{O}_{2}$ and $\mathrm{O}_{2}{ }^{-}$in the L2, L4, L6 and WT leaves were analyzed by DAB (A) and NBT (B) staining. (C-E) Determination of SOD, POD and CAT activity. The value is the mean \pm SD of three biological replicates. ${ }^{* *}$ Indicates significant differences between the L2/L4/L6 plants and the WT plants. ${ }^{* *}$ Indicates $p<0.01$. 
A

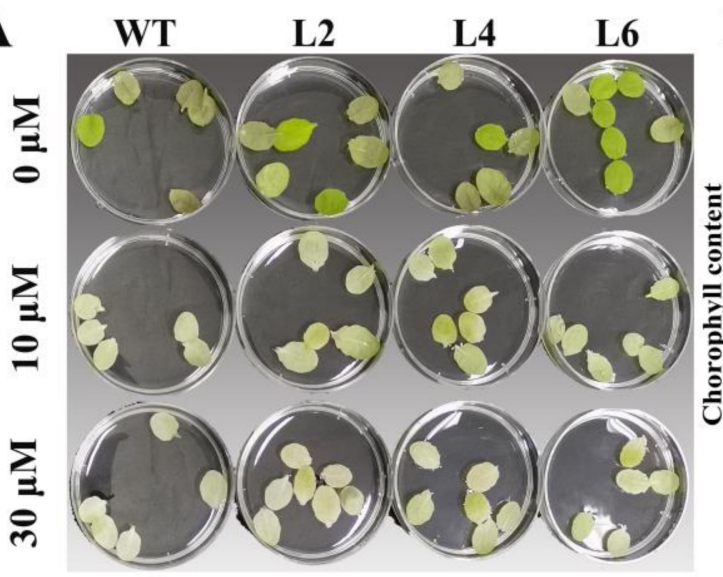

B

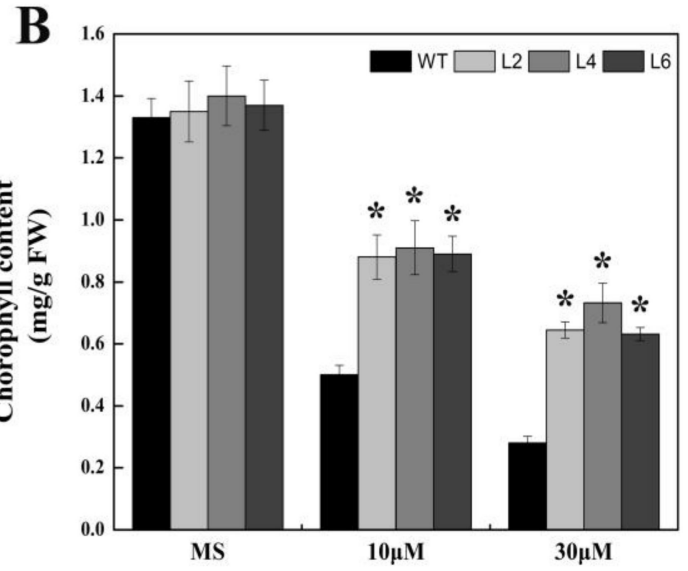

Figure 8. Comparison of the oxidative stress tolerance between the WT and the transgenic plant leaves. (A) The leaves of three-week-old L2, L4, L6 and WT Arabidopsis were treated with methyl viologen (MV) at concentrations of 0,10 and $30 \mu \mathrm{M}$ for $24 \mathrm{~h}$. (B) Chlorophyll content in the leaves after stress treatment. Each value is based on three biological replicates, and the error bars represent $\pm \mathrm{SD}$. *Indicates significance compared with the WT $(p<0.05)$.

\subsection{Ectopic Expression of FtbZIP5 Regulated Downstream Stress Response Genes}

To study the molecular mechanism of FtbZIP5 regulation of stress response-related genes, qRT-PCR was used to detect the expression levels of ABA-dependent stress response genes, including RD29A, $R D 29 B, R A B 18, R D 26, R D 20$ and COR15, in Arabidopsis under drought or salt stress. The expression level of the transgenic lines (L1, L3 and L5) and WT plants without treatment was used as the corresponding control, and as shown in Figure 9, there was no remarkable change. However, after the drought or salt stress treatment, the expression of these stress response genes increased significantly. Thus, we speculate that FtbZIP5 might regulate stress-related genes through the ABA signaling pathway to increase plant resistance to drought exposure and high salt levels.

\subsection{Ectopic Expression of FtbZIP5 Partially Recovered the Insensitivity of the Mutant abi5-1 to ABA}

To determine whether FtbZIP5 has biological significance in the ABA signaling pathway in the plants, we constructed abi5-1 transgenic lines from pCAMBIA1305-FtbZIP5 (abi5-1::FtbZIP5). Seed germination of FtbZIP5-overexpressed Arabidopsis lines (WT::FtbZIP5), WT, mutant abi5-1 and abi5-1 were compared by $0 \mu \mathrm{M}$ ABA and $0.4 \mu \mathrm{M}$ ABA treatment. As shown in Figure 10A and 10D, the germination rates of the different types of seeds were basically the same on the 7th day when none were treated with ABA. When $0.4 \mu \mathrm{M}$ ABA treatment was used, the germination time and rate of the mutant abi5-1 seeds were similar to those under normal conditions, and the germination time and rate were inhibited by ABA in the other seeds (Figure 10B,E). Surprisingly, the seed germination of the FtbZIP5-overexpressed lines (WT::FtbZIP5) was inhibited most obviously, and the seeds of the WT and abi5-1 transgenic lines were moderately inhibited. Our results revealed that FtbZIP5 was able to partially compensate for mutant abi5-1 during seed germination. 

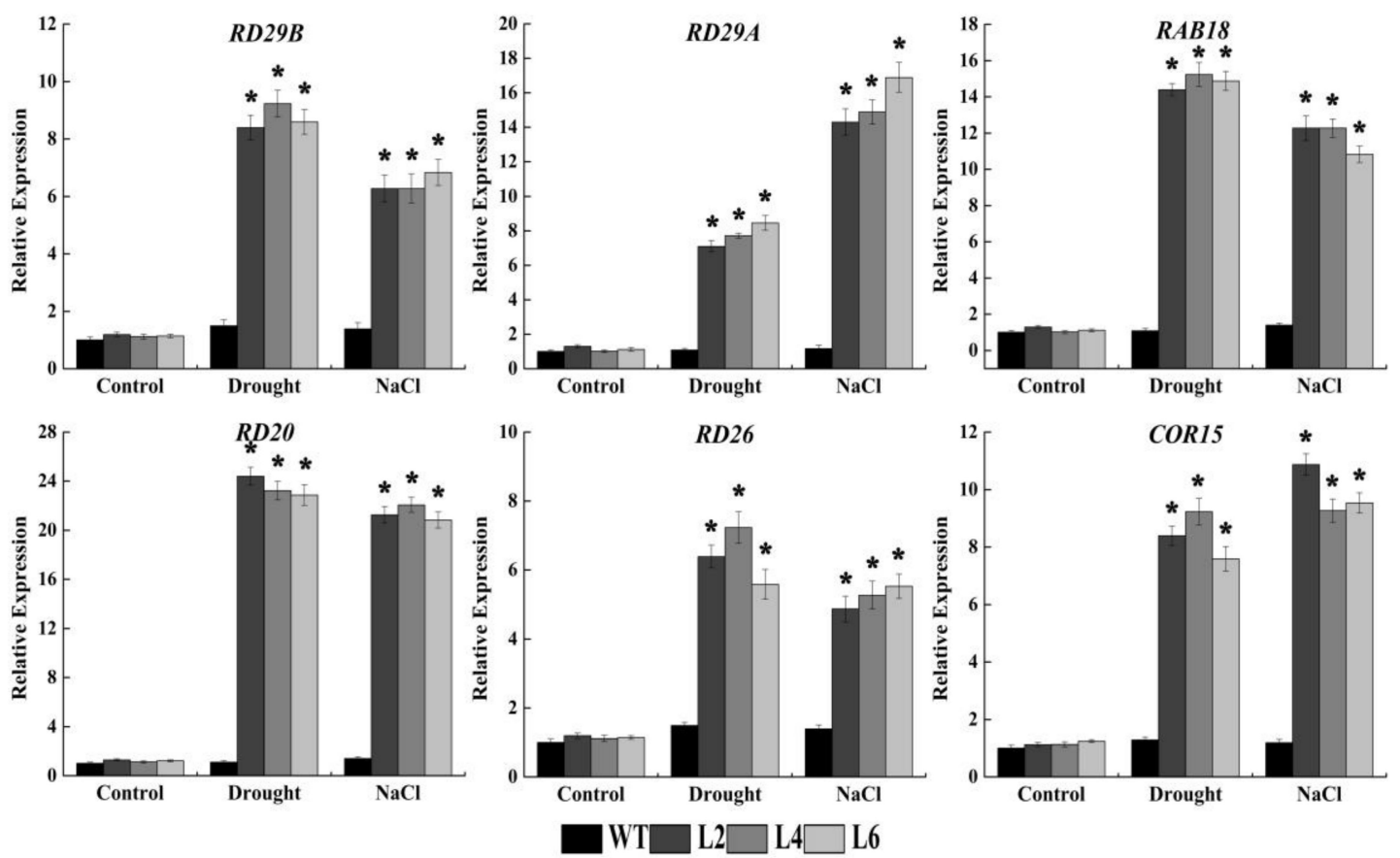

Figure 9. Analysis of ABA-dependent stress-related gene expression levels in transgenic lines and WT. Three-week-old Arabidopsis seedlings were treated without water and with $200 \mathrm{mM} \mathrm{NaCl}$ for one week until the RNA was extracted. The expression level of the WT under normal growth conditions was used as a control. The error bar represents the standard deviation between the three biological replicates. * Indicates that the expression level of the gene in the transgenic lines was significantly different from that in the WT. Significance was based on Student's t-test $(p<0.05)$.

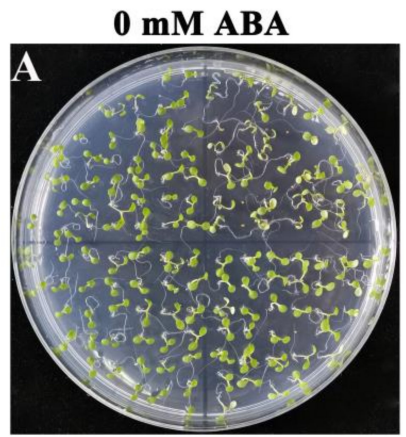

D

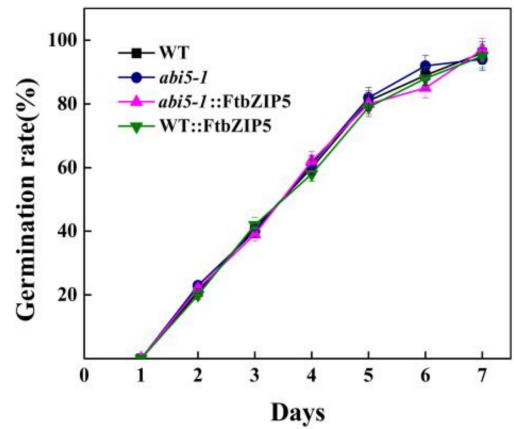

$0.4 \mathrm{mM}$ ABA

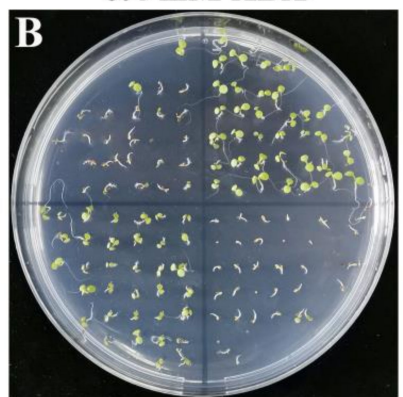

C

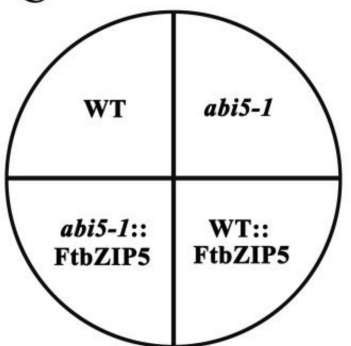

$\mathbf{E}$

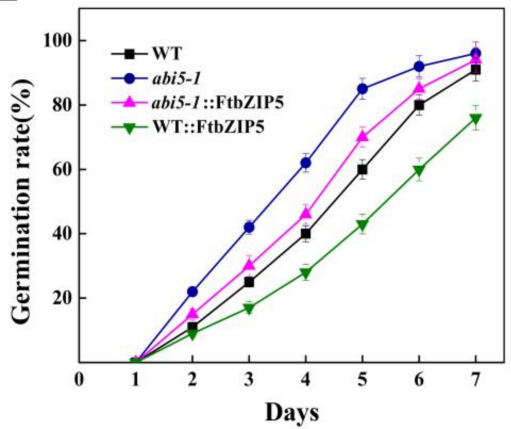

Figure 10. Ectopic expression of FtbZIP5 increases the ABA sensitivity of abi5-1 in Arabidopsis during seed germination. (A,B) The seeds of the WT, abi5-1, abi5-1:: FtbZIP5 and WT:: FtbZIP5 plants were cultured on 1/2-strength MS medium with $0 \mu \mathrm{M}$ ABA or $0.4 \mu \mathrm{M}$ ABA for 7 days. (C) A schematic map showing the four areas sown on the medium. $(\mathbf{D}, \mathbf{E})$ The germination rate of the seeds was calculated. 


\subsection{The Physical Interaction between FtbZIP5 and SnRK2.6 Proteins in Yeast}

Based on three members of the third subclass of FtSnRK2 protein kinases obtained from tartary buckwheat by previous studies [23], the interaction analysis was carried out by a yeast two-hybrid experiment. Both the negative control and experimental transformants could grow normally on the double-deficient medium. However, only the FtSnRK2.6 + FtbZIP5 yeast strain survived in the four deficient media and showed blue color indicative of $x-\beta-G a l$ on filter paper (Figure 11). Collectively, these data allowed us to conclude that FtbZIP5 and FtSnRK2.6 can interact with each other.

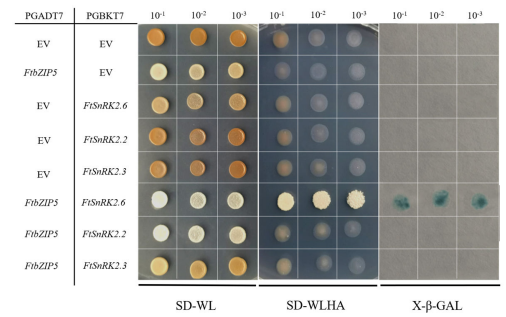

Figure 11. Y2H experiment used to analyze the relationship between FtSnRK2.6/2.3/2.2 and FtbZIP5. The combination plasmid transformed in AH109 yeast cells were serially diluted 10-, 100- and 1000-fold, cultured on SD/-Trp-Leu medium and SD/-Trp-\Leu-His-Ade medium, respectively, and then stained with $x$ - $\beta$-gal.

\section{Discussion}

In this study, we identified a novel stress-resistant AREB/ABF transcription factor gene, FtbZIP5, from tartary buckwheat. Similar to other studies, the protein is located in the nucleus, has transcriptional activation activity and can specifically bind to ABRE cis-acting elements. Similar to other studies, the protein is located in the nucleus and has transcriptional activation activity. The results from the bioinformatics analysis showed that FtbZIP5 has a high affinity for ABFs related to stress in Arabidopsis and had four possible phosphorylation sites. Additionally, the results from the expression analysis showed that FtbZIP5 was significantly induced by drought, salt and exogenous ABA. Further studies showed that the ectopic expression of FtbZIP5 could regulate stress-induced genes through an ABA-dependent signaling pathway and enhance drought and salt tolerance in transgenic Arabidopsis by increasing SOD, POD and CAT levels, thereby reducing the ROS content of transgenic Arabidopsis. Furthermore, the ectopic expression of FtbZIP5 can increase the antioxidant activity and sensitivity of transgenic plants to exogenous ABA and partially restored the insensitivity of mutant abi5-1 to ABA. Additionally, we also screened FtSnRK2.6, which may phosphorylate FtbZIP5. Based on the above results, we propose a diagram showing the role of FtbZIP5 in the stress response regulation mechanism (Figure 12). This study can be used to advance the understanding of the tartary buckwheat stress resistance mechanism and enrich the research into AREB/ABFs in plants.

Previous studies have demonstrated that AREB/ABFs, as positive regulators of the ABA signaling pathway, play important roles in osmotic stress regulation in plants [24]. For example, overexpression of OSBZIP72 from rice enhanced the drought resistance and ABA sensitivity of transgenic plants [25]. The wheat TaAREB3 transcription factor improved the drought and freezing tolerance in Arabidopsis [26], and overexpression of GhABF2 enhanced the salt and drought tolerance of cotton [27]. In Arabidopsis, the homologous protein $\mathrm{ABI} 5$ of the $\mathrm{AREB} / \mathrm{ABF}$ family is the central regulator of $\mathrm{ABA}$ signal transduction [28,29]. Previous studies have shown that mutants of ABI5 and members of the AREB/ABF family reduce the insensitivity to exogenous ABA during seed germination [30,31]. Our results showed that the ectopic expression of FtbZIP5 increased the sensitivity of mutant abi5-1 to ABA. However, the ectopic expression of FtbZIP5 could not make the abi5-1 mutant reach the same sensitivity to ABA as the WT. Similar to the findings from previous studies, the functions of ABI5 and AREB/ABFs are redundant, and ABI5 plays a major regulatory role within 10 days of seed germination [32].

It is well known that plants can undergo a series of physiological and biochemical adjustments in vivo to adapt to adverse living environments. Reactive oxygen species (ROS) accumulate in 
plants under abiotic stress, and excessive ROS can cause oxidative damage to cell components [33,34]. Plant scavenging enzyme (SOD, POD and CAT) systems can remove redundant ROS, thereby increasing plant adaptability [35]. Malondialdehyde (MDA) is the most important product of membrane lipid peroxidation [36]. Proline acts as an osmotic regulator in plant cytoplasm [37]. Both MDA and proline are main indicators for determining plant stress resistance. Previous studies have shown that AREB/ABFs, such as ABP9 and VvABF1, can increase antioxidant enzyme activity and proline content, and reduce ROS accumulation and MDA content to improve the resistance of transgenic plants [38,39]. As expected, our experimental results also show that FtbZIP5 has functions similar to those of these AREB/ABFs. Additionally, after activation of the AREB/ABF-SnRK2 signaling pathway, AREB/ABF transcription factors directly interact with ABRE elements in the promoter region of downstream stress response genes [40]. It has been shown that these six stress/ABA-induced genes (RD29A, $R D 29 B, R A B 18, R D 26, R D 20$ and COR15) are directly regulated by ABRE/ABFs in Arabidopsis [41,42]. The results of yeast one-hybrid experiment also showed that FtbZIP5 could specifically bind to ABRE cis-acting element.

Phosphorylation, acetylation, methylation and other post-translational modifications can integrate multiple signals at the same level [21]. In Arabidopsis, studies have revealed that triple mutants of snrk2.6, snrk2.3 and snrk2.2 control ABA-activated phosphorylation of AREB/ABFs [43]. Interestingly, subsequent study found that AREB/ABFs were phosphorylated by multiple protein kinases of the SnRK2 family [44]. Recent reports have also suggested that the response of plant cells to salt stress, drought stress and stress hormone ABA-induced signaling depends largely on the SnRK protein kinase family [45]. Our yeast two hybrid experiment showed that there was interaction between FtbZIP5 and the homologous protein FtSnRK2.6 of SnRK2.6, but their phosphorylation requires further verification. Similarly, in apple, MdAREB2 is phosphorylated by the SnRK2 homologous protein MdCIPK22 [46]. Previous studies have found an interaction between FtbZIP83 and FtSnRK2.6/2.3 [23]. The differences in their interaction may be caused by the complexity of AREB/ABF transcription factor function and the diversity of their post-transcriptional modifications. Collectively, the data suggest that the SnRK2-AREB/ABF signaling pathway plays a pivotal role in the cross-talk of that is induced by abiotic stress signaling in plants.

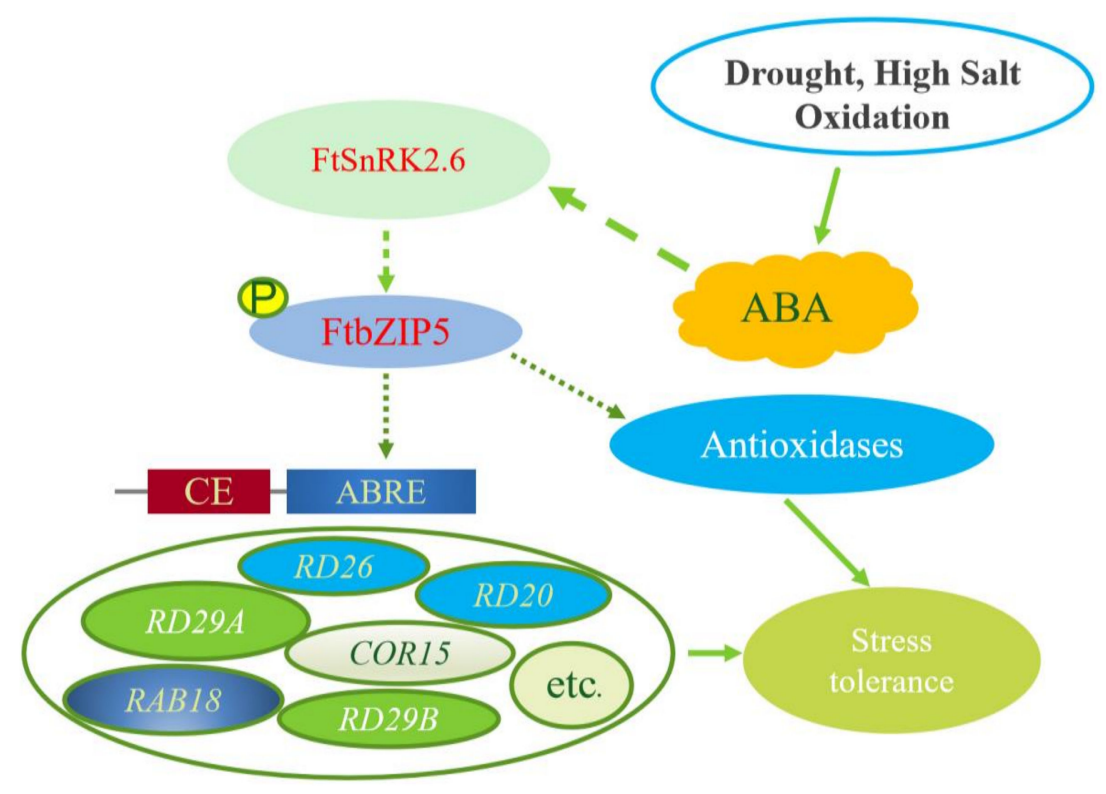

Figure 12. FtbZIP5 of Fagopyrum tataricum is involved in the ABA-dependent signaling pathway and may improve the stress tolerance of plants via the phosphorylation of FtSnRK2.6. Dotted and dashed lines indicate that the relationship between the two is not directly proved, while complete arrow lines indicate that it has been confirmed. 


\section{Materials and Methods}

\subsection{Cloning of FtbZIP5 Gene, Construction of the Vector and Transformation of Arabidopsis Thaliana}

To construct the fusion protein expression vector pCHF-FtbZIP5-YFP, a pair of specific primers were designed to amplify the cDNA sequence of FtbZIP5. The CDS of FtbZIP5 was cloned into the plant expression vector pCHF-YFP by BamHI and EcoRI.

The positive Agrobacterium contained 35S:FtbZIP5-YFP, which was transferred into WT Arabidopsis through the floral dip transformation method [47]. Then, the transgenic homozygous cell lines were screened by 1/2-strength MS medium containing $50 \mathrm{mg} / \mathrm{mL}$ kanamycin and confirmed at the molecular level by PCR. For transcriptional activation experiments, the CDS of FtbZIP5 was inserted into the pBridge vector to generate pBridge-FtbZIP5 using the two restriction sites: EcoRI and SalI. In the yeast one hybrid assay, the three tandem copy sequences of ABRE (ACGTG) and mABRE (AAAAA) were inserted into pHIS2.1 vector of EcoRI and BamHI sites, respectively. Using NdeI and EcoRI as restriction sites, the CDS of FtbZIP5 was cloned into the vector pGADT7. For the functional replenishment mutant experiment, we constructed a vector, pCAMBIA1305-FtbZIP5, that was transferred into Arabidopsis mutant abi5-1 via Agrobacterium-mediated transformation. The homozygous transgenic lines of generation T3 were obtained by the methods described above. The primers are presented in Supplementary Table S1.

The phylogenetic tree was constructed using the neighbor-joining method with Mega 7.0 software, and DNAMAN software was used for multiple sequence alignment. The physicochemical properties of FtbZIP5 protein were obtained through ProtParam.

\subsection{Plant Materials and Stress Treatments}

The tartary buckwheat seeds (Xiqiao No. 2) used in this study were provided by the Academy of Agricultural Sciences (Xichang, China). The seeds were germinated on wet filter paper and then transferred to Hoagland solution for culturing. For the stress treatment, 2-week-old seedlings with similar growth and development were treated with $100 \mu \mathrm{M} \mathrm{ABA}, 30 \%$ PEG and $150 \mathrm{mM} \mathrm{NaCl}$. Samples were collected at $0,1,1.5,2,3,6$ and $10 \mathrm{~h}$ of treatment within a photoperiod and then stored immediately at $-80^{\circ} \mathrm{C}$ until the RNA was extracted.

For the seed germination experiments, sterilized WT and transgenic seeds were germinated on 1/2-strength MS agar plates containing $0.6 \mu \mathrm{M}$ ABA, $150 \mathrm{mM} \mathrm{NaCl}$ and $200 \mathrm{mM}$ mannitol. The germination rate of the seeds was recorded every $24 \mathrm{~h}$ for 7 days. For the root length experiments, seedlings aged 3 days and growing in 1/2-strength MS media were transplanted to the same stress plate as described above, and the root length was measured on the 7 th day. The complement mutant experiment was carried out with $0.4 \mu \mathrm{M} \mathrm{ABA}$, which was similar to that used in the seed germination experiment.

For the drought and salt stress experiments, the T3 homozygous transgenic lines and WT plants were cultivated in soil for three weeks under the same growth conditions. For the drought treatment, 3-week-old seedlings were denied water for 2 weeks and then were rewatered to determine the survival rate 7 days after the rewatering was initiated. For the salt treatment, 3-week-old seedlings were treated with $200 \mathrm{mM} \mathrm{NaCl}$ for 3 weeks, and their phenotypic changes were recorded every 7 days.

For the oxidative stress experiment, the leaves from the 4-week-old transgenic lines and the WT were treated with 1/2-strength MS containing $10 \mu \mathrm{M}$ or $30 \mu \mathrm{M}$ methyl viologen, respectively. After $48 \mathrm{~h}$, the degree of chlorosis was observed, and the chlorophyll content was determined according to the Heath et al. method [48]. The abovementioned growth conditions were generated with a white fluorescent lamp $\left(14 \mathrm{~h} / 10 \mathrm{~h}\right.$, light/dark) at room temperature of $23 \pm 1{ }^{\circ} \mathrm{C}$ with relative humidity of $50 \%-60 \%$.

\subsection{RNA Extraction and qRT-PCR Analysis}

Total RNA from mixed tissues of the plant seedlings was extracted using an EASYSpin Plant RNA kit (Aidlab, Beijing, China), and the first-strand cDNA was synthesized by being reverse transcribed with 
a HiScript II $1^{\text {st }}$ strand cDNA synthesis kit (Vazyme Biotech, Nanjing, China). The RT-PCR amplification enzyme (TB Green Premix Ex TapII), primer and template were mixed and amplified in a CFX96-RT-PCR machine. Three biological replicates were generated for each sample, and the results were calculated by the $2^{-\Delta \Delta C T}$ method. The primers used for qRT-PCR are listed in Supplementary Table S2.

\subsection{Subcellular Localization, Transcriptional Activation and Binding with ABRE Elements of FtbZIP5}

For the localization study, the FtbZIP5:YFP fusion protein was selected for transformation into Arabidopsis using a method similar to that of previous studies [49]. The sterilized seeds of transgenic Arabidopsis were cultured in 1/2-strength MS for two weeks. DAPI, as a marker of nuclear positioning, emits blue fluorescence under blue excitation light. The results were obtained by observing the mature zone of the roots with yellow and blue excitation light in confocal laser microscopy. For the transcriptional activation assays, pBridge-FtbZIP5, pBridge-FtbHLH2 and pBridge (negative control) were transformed into yeast AH109. A galactosidase activity assay was performed on a filter paper by selecting transformants that normally grow in SD/-His-Trp media. For yeast one hybrid experiment, pGADT7-FtbZIP5 + pHIS2.1-3×ABRE/pHIS2.1-3×mABRE/pHIS2.1 vector combinations were respectively transformed into yeast Y187. After 2-3 days of SD/-Trp-Leu culture, the growth was observed on the SD/-Trp-Leu-His medium containing $50 \mathrm{mM}$ 3-AT.

\subsection{Measurement of Antioxidant Enzymes, Proline and Malondialdehyde}

To further understand the function of FtbZIP5 under drought/salt treatment, we measured the activity of three antioxidant enzymes (SOD, POD and CAT) and the Pro and MDA content. After two days of drought and salt treatment, the activity levels of SOD, POD and CAT in the leaves were determined by the method described previously [32]. For the determination of Pro (proline) and MDA (malondialdehyde) content, the transgenic lines and the WT were selected one week after exposure to drought/salt stress, as described previously [50].

\subsection{Histochemical Staining with $D A B$ and NBT}

To evaluate the extent of $\operatorname{ROS}\left(\mathrm{H}_{2} \mathrm{O}_{2}\right.$ and $\left.\mathrm{O}_{2}{ }^{-}\right)$accumulation in transgenic lines and the WT after drought and salt stress, $\mathrm{DAB}$ and NBT histochemical staining were used. After two weeks of stress treatment, the leaves were stained with NBT $(0.5 \mathrm{mg} / \mathrm{mL})$ for $3 \mathrm{~h}$ and DAB $(1 \mathrm{mg} / \mathrm{mL})$ for $8 \mathrm{~h}$ under dark conditions. Then, the stained leaves were boiled in $80 \%$ ethanol until the green was completely faded.

\section{7. $Y 2 H$ Assay}

Yeast two-hybrid (Y2H) analysis was carried out to determine whether FtbZIP5 protein could interact with FtSnRK2.6/2.3/2.2 kinase. The coding sequences of FtbZIP5, FtSnRK2.6, FtSnRK2.3 and FtSnRK2.2 were cloned into pGADT7 or pGBKT7 vectors, and FtSnRK2.6/2.3/2.2 + FtbZIP5 in the experimental group and FtSnRK2.6/2.3/2.2 + pGADT7, pGBKT7 + FtbZIP5 and pGBKT7 + pGADT7 in the negative control group were established. Next, the recombinant plasmids in the above combinations were transferred into yeast AH109. The specific designed primers are shown in Supplementary Table S3. The positive transformants were diluted 0.1-, 0.01- and 0.001-fold, respectively, and then cultured on SD/-Trp-Leu and SD/-Leu-Trp-His-Ade selective media at $28^{\circ} \mathrm{C}$ for 3 days. In the last step, yeast normally growing in the four deficient media were stained with $\mathrm{X}$ - $\beta$-gal on filter paper.

Supplementary Materials: Supplementary materials can be found at http://www.mdpi.com/1422-0067/21/3/1123/ s1.

Author Contributions: Conceptualization, Q.L. and Q.W.; Data curation, Q.L.; Formal analysis, Q.L.; Funding acquisition, Q.W.; Investigation B.L. and J.K.; Methodology, X.W. and Q.D.; Project administration, H.Z.; Resources, Q.L.; Software, Q.L. and C.L.; Supervision, H.C.; Writing - original draft, Q.L.; Writing - review \& editing, Q.L. and H.Z. All authors have read and agreed to the published version of the manuscript.

Funding: This research did not receive any specific grant from funding agencies in the public, commercial or not-for-profit sectors. 
Conflicts of Interest: The authors declare no conflict of interest.

\section{References}

1. Nakashima, K.; Ito, Y.; Yamaguchishinozaki, K. Transcriptional Regulatory Networks in Response to Abiotic Stresses in Arabidopsis and Grasses. Plant Physiol. 2009, 149, 88-95. [CrossRef] [PubMed]

2. Cutler, S.R.; Rodriguez, P.L.; Finkelstein, R.R.; Abrams, S.R. Abscisic Acid: Emergence of a Core Signaling Network. Annu. Rev. Plant Biol. 2010, 61, 651-679. [CrossRef] [PubMed]

3. Raghavendra, A.S.; Gonugunta, V.K.; Christmann, A.; Grill, E. ABA perception and signaling. Trends Plant Sci. 2010, 15, 395-401. [CrossRef] [PubMed]

4. Finkelstein, R.R.; Gampala, S.S.L.; Rock, C.D. Abscisic Acid Signaling in Seeds and Seedlings. Plant Cell 2002, 14, 15. [CrossRef] [PubMed]

5. Yamaguchishinozaki, K.; Shinozaki, K. Transcriptional regulatory networks in cellular responses and tolerance to dehydration and cold stresses. Plant Biol. 2006, 57, 781-803. [CrossRef] [PubMed]

6. Fujita, Y.; Fujita, M.; Shinozaki, K.; Yamaguchishinozaki, K. ABA-mediated transcriptional regulation in response to osmotic stress in plants. J. Plant Res. 2011, 124, 509-525. [CrossRef]

7. Drogelaser, W.; Snoek, L.B.; Snel, B.; Weiste, C. The Arabidopsis bZIP transcription factor family-An update. Curr. Opin. Plant Biol. 2018, 45, 36-49. [CrossRef]

8. Kim, S.Y.; Ma, J.; Perret, P.; Li, Z.; Thomas, T.L. Arabidopsis ABI5 Subfamily Members Have Distinct DNA-Binding and Transcriptional Activities. Plant Physiol. 2002, 130, 688-697. [CrossRef]

9. Bensmihen, S.; Giraudat, J.; Parcy, F. Characterization of three homologous basic leucine zipper transcription factors (bZIP) of the ABI5 family during Arabidopsis thaliana embryo maturation. J. Exp. Bot. 2005, 56, 597-603. [CrossRef]

10. Bensmihen, S.; Rippa, S.; Lambert, G.; Jublot, D.; Pautot, V.; Granier, F.; Giraudat, J.; Parcy, F. The Homologous ABI5 and EEL Transcription Factors Function Antagonistically to Fine-Tune Gene Expression during Late Embryogenesis. Plant Cell 2002, 14, 1391-1403. [CrossRef]

11. Kim, S.Y. The role of ABF family bZIP class transcription factors in stress response. Physiol. Plant. 2005, 126, 519-527. [CrossRef]

12. Lopezmolina, L.; Chua, N. A Null Mutation in a bZIP Factor Confers ABA-Insensitivity in Arabidopsis thaliana. Plant Cell Physiol. 2000, 41, 541-547. [CrossRef] [PubMed]

13. Suzuki, M.; Ketterling, M.G.; Li, Q.; Mccarty, D.R. Viviparous1 Alters Global Gene Expression Patterns through Regulation of Abscisic Acid Signaling. Plant Physiol. 2003, 132, 1664-1677. [CrossRef] [PubMed]

14. Uno, Y.; Furihata, T.; Abe, H.; Yoshida, R.; Shinozaki, K.; Yamaguchishinozaki, K. Arabidopsis basic leucine zipper transcription factors involved in an abscisic acid-dependent signal transduction pathway under drought and high-salinity conditions. Proc. Natl. Acad. Sci. USA 2000, 97, 11632-11637. [CrossRef] [PubMed]

15. Choi, H.; Hong, J.; Ha, J.; Kang, J.; Kim, S.Y. ABFs, a Family of ABA-responsive Element Binding Factors. J. Biol. Chem. 2000, 275, 1723-1730. [CrossRef]

16. Yoshida, T.; Fujita, Y.; Sayama, H.; Kidokoro, S.; Maruyama, K.; Mizoi, J.; Shinozaki, K.; Yamaguchishinozaki, K. AREB1, AREB2, and ABF3 are master transcription factors that cooperatively regulate ABRE-dependent ABA signaling involved in drought stress tolerance and require ABA for full activation. Plant J. 2010, 61, 672-685. [CrossRef]

17. Finkelstein, R.R.; Lynch, T.J. The Arabidopsis Abscisic Acid Response Gene ABI5 Encodes a Basic Leucine Zipper Transcription Factor. Plant Cell 2000, 12, 599-609. [CrossRef]

18. Zhu, S.; Yu, X.; Wang, X.; Zhao, R.; Li, Y.; Fan, R.; Shang, Y.; Du, S.; Wang, X.; Wu, F. Two Calcium-Dependent Protein Kinases, CPK4 and CPK11, Regulate Abscisic Acid Signal Transduction in Arabidopsis. Plant Cell 2007, 19, 3019-3036. [CrossRef]

19. Kagaya, Y.; Hobo, T.; Murata, M.; Ban, A.; Hattori, T. Abscisic acid-induced transcription is mediated by phosphorylation of an abscisic acid response element binding factor, TRAB1. Plant Cell 2002, 14, 3177-3189. [CrossRef]

20. Yoshida, T.; Fujita, Y.; Maruyama, K.; Mogami, J.; Todaka, D.; Shinozaki, K.; Yamaguchishinozaki, K. Four Arabidopsis AREB/ABF transcription factors function predominantly in gene expression downstream of SnRK2 kinases in abscisic acid signalling in response to osmotic stress. Plant Cell Environ. 2015, 38, 35-49. [CrossRef] 
21. Fujita, Y.; Nakashima, K.; Yoshida, T.; Katagiri, T.; Kidokoro, S.; Kanamori, N.; Umezawa, T.; Fujita, M.; Maruyama, K.; Ishiyama, K. Three SnRK2 Protein Kinases are the Main Positive Regulators of Abscisic Acid Signaling in Response to Water Stress in Arabidopsis. Plant Cell Physiol. 2009, 50, 2123-2132. [CrossRef] [PubMed]

22. Correa, L.G.G.; Rianopachon, D.M.; Schrago, C.G.; Santos, R.V.D.; Muellerroeber, B.; Vincentz, M. The role of bZIP transcription factors in green plant evolution: Adaptive features emerging from four founder genes. PLoS ONE 2008, 3, e2944. [CrossRef] [PubMed]

23. Li, Q.; Wu, Q.; Wang, A.; Lv, B.; Dong, Q.; Yao, Y.; Wu, Q.; Zhao, H.; Li, C.; Chen, H.; et al. Tartary buckwheat transcription factor FtbZIP83 improves the drought/salt tolerance of Arabidopsis via an ABA-mediated pathway. Plant Physiol. Biochem. PPB 2019, 144, 312-323. [CrossRef] [PubMed]

24. Li, C.; Yue, J.; Wu, X.; Xu, C.; Yu, J. An ABA-responsive DRE-binding protein gene from Setaria italica, SiARDP, the target gene of SiAREB, plays a critical role under drought stress. J. Exp. Bot. 2014, 65, 5415-5427. [CrossRef]

25. Lu, G.; Gao, C.; Zheng, X.; Han, B. Identification of OsbZIP72 as a positive regulator of ABA response and drought tolerance in rice. Planta 2009, 229, 605-615. [CrossRef]

26. Wang, J.; Li, Q.; Mao, X.; Li, A.; Jing, R. Wheat Transcription Factor TaAREB3 Participates in Drought and Freezing Tolerances in Arabidopsis. Int. J. Biol. Sci. 2016, 12, 257-269. [CrossRef]

27. Liang, C.; Meng, Z.; Meng, Z.; Malik, W.; Yan, R.; Lwin, K.M.; Lin, F.; Wang, Y.X.; Sun, G.; Zhou, T. GhABF2, a bZIP transcription factor, confers drought and salinity tolerance in cotton (Gossypium hirsutum L.). Sci. Rep. 2016, 6, 35040. [CrossRef]

28. Stone, S.L.; Williams, L.A.; Farmer, L.M.; Vierstra, R.D.; Callis, J. KEEP ON GOING, a RING E3 Ligase Essential for Arabidopsis Growth and Development, Is Involved in Abscisic Acid Signaling. Plant Cell 2006, 18, 3415-3428. [CrossRef]

29. Wang, Y.; Li, L.; Ye, T.; Lu, Y.; Chen, X.; Wu, Y. The inhibitory effect of ABA on floral transition is mediated by ABI5 in Arabidopsis. J. Exp. Bot. 2013, 64, 675-684. [CrossRef]

30. Brocard, I.M.; Lynch, T.J.; Finkelstein, R.R. Regulation and Role of the Arabidopsis Abscisic Acid-Insensitive 5 Gene in Abscisic Acid, Sugar, and Stress Response. Plant Physiol. 2002, 129, 1533-1543. [CrossRef]

31. Skubacz, A.; Daszkowskagolec, A.; Szarejko, I. The Role and Regulation of ABI5 (ABA-Insensitive 5) in Plant Development, Abiotic Stress Responses and Phytohormone Crosstalk. Front. Plant Sci. 2016, 7, 1884. [CrossRef] [PubMed]

32. Finkelstein, R.R.; Gampala, S.S.L.; Lynch, T.J.; Thomas, T.L.; Rock, C.D. Redundant and distinct functions of the ABA response loci ABA- INSENSITIVE(ABI)5 and ABRE-BINDING FACTOR (ABF)3. Plant Mol. Biol. 2005, 59, 253-267. [CrossRef] [PubMed]

33. Liu, Y.; Ye, N.; Liu, R.; Chen, M.; Zhang, J. H2O2 mediates the regulation of ABA catabolism and GA biosynthesis in Arabidopsis seed dormancy and germination. J. Exp. Bot. 2010, 61, 2979-2990. [CrossRef] [PubMed]

34. Zhang, X.; Wang, L.; Meng, H.; Wen, H.; Fan, Y.; Zhao, J. Maize ABP9 enhances tolerance to multiple stresses in transgenic Arabidopsis by modulating ABA signaling and cellular levels of reactive oxygen species. Plant Mol. Biol. 2011, 75, 365-378. [CrossRef]

35. Environment and plant metabolism: Flexibility and acclimation. In Environment $\mathcal{E}$ Plant Metabolism Flexibility $\mathcal{E}$ Acclimation XVI + P; Smirnoff, N. (Ed.) Bios Scientific Publishers: Oxford, UK, 1995; Volume 7, pp. 405-410.

36. Tu, M.; Wang, X.; Huang, L.; Guo, R.; Zhang, H.; Cai, J.; Wang, X. Expression of a grape bZIP transcription factor, VqbZIP39, in transgenic Arabidopsis thaliana confers tolerance of multiple abiotic stresses. Plant Cell Tissue Organ Cult. 2016, 125, 537-551. [CrossRef]

37. Rejeb, K.B.; Abdelly, C.; Savoure, A. How reactive oxygen species and proline face stress together. Plant Physiol. Biochem. 2014, 80, 278-284. [CrossRef]

38. Liu, J.; Chu, J.; Ma, C.; Jiang, Y.; Ma, Y.; Xiong, J.; Cheng, Z. Overexpression of an ABA-dependent grapevine bZIP transcription factor, VvABF2, enhances osmotic stress in Arabidopsis. Plant Cell Rep. 2019, 38, 587-596. [CrossRef]

39. Wang, C.; Lu, G.; Hao, Y.; Guo, H.; Guo, Y.; Zhao, J.; Cheng, H. ABP9, a maize bZIP transcription factor, enhances tolerance to salt and drought in transgenic cotton. Planta 2017, 246, 453-469. [CrossRef] 
40. Fujita, Y.; Yoshida, T.; Yamaguchishinozaki, K. Pivotal role of the AREB/ABF-SnRK2 pathway in ABRE-mediated transcription in response to osmotic stress in plants. Physiol. Plant. 2013, 147, 15-27. [CrossRef]

41. Hobo, T.; Kowyama, Y.; Hattori, T. A bZIP factor, TRAB1, interacts with VP1 and mediates abscisic acid-induced transcription. Proc. Natl. Acad. Sci. USA 1999, 96, 15348-15353. [CrossRef]

42. Takahashi, S.; Katagiri, T.; Yamaguchishinozaki, K.; Shinozaki, K. An Arabidopsis Gene Encoding a Ca2+-Binding Protein is Induced by Abscisic Acid during Dehydration. Plant Cell Physiol. 2000, 41, 898-903. [CrossRef] [PubMed]

43. Garcia, M.N.M.; Giammaria, V.; Grandellis, C.; Tellezinon, M.T.; Ulloa, R.M.; Capiati, D.A. Characterization of StABF1, a stress-responsive bZIP transcription factor from Solanum tuberosum L. that is phosphorylated by StCDPK2 in vitro. Planta 2012, 235, 761-778. [CrossRef] [PubMed]

44. Fujita, M.; Fujita, Y.; Noutoshi, Y.; Takahashi, F.; Narusaka, Y.; Yamaguchishinozaki, K.; Shinozaki, K. Crosstalk between abiotic and biotic stress responses: A current view from the points of convergence in the stress signaling networks. Curr. Opin. Plant Biol. 2006, 9, 436-442. [CrossRef] [PubMed]

45. Zhu, J. Abiotic Stress Signaling and Responses in Plants. Cell 2016, 167, 313-324. [CrossRef]

46. Ma, Q.; Sun, M.; Lu, J.; Liu, Y.; You, C.; Hao, Y. An apple CIPK protein kinase targets a novel residue of AREB transcription factor for ABA-dependent phosphorylation. Plant Cell Environ. 2017, 40, 2207-2219. [CrossRef]

47. Bechtold, N.; Pelletier, G. In planta Agrobacterium mediated transformation of adult Arabidopsis thaliana plants by vacuum infifiltration. Methods Mol. Biol. 1998, 82, 259-266.

48. Heath, R.L.; Packer, L. Photoperoxidation in isolated chloroplasts. I. Kinetics and stoichiometry of fatty acid peroxidation. Arch. Biochem. Biophys. 1968, 125, 189-198. [CrossRef]

49. Zhao, J.; Zhang, W.; Zhao, Y.; Gong, X.; Guo, L.; Zhu, G.; Wang, X.; Gong, Z.; Schumaker, K.S.; Guo, Y. SAD2, an Importin $\beta$-Like Protein, Is Required for UV-B Response in Arabidopsis by Mediating MYB4 Nuclear Trafficking. Plant Cell 2007, 19, 3805-3818. [CrossRef]

50. Jaffar, M.A.; Song, A.; Faheem, M.; Chen, S.; Jiang, J.; Liu, C.; Fan, Q.; Chen, F. Involvement of CmWRKY10 in Drought Tolerance of Chrysanthemum through the ABA-Signaling Pathway. Int. J. Mol. Sci. 2016, 17, 693. [CrossRef]

(C) 2020 by the authors. Licensee MDPI, Basel, Switzerland. This article is an open access article distributed under the terms and conditions of the Creative Commons Attribution (CC BY) license (http://creativecommons.org/licenses/by/4.0/). 